\title{
„Der Kasuar - ein Christ in der Industriegesellschaft"
}

\author{
von Hermann-Josef Spital
}

1. Der zuerst 1979 im Styria-Verlag erschienene Roman von Matthias Mander: „Der Kasuar“ ist singulär. Ich kenne kein anderes Buch, mit dem ich diesen Roman vergleichen könnte. 1982 ist das 419 Seiten starke Buch auch als Taschenbuch im Ullstein-Verlag erschienen; daraus darf man folgern, daß es sich durchzusetzen beginnt. Das ist keineswegs selbstverständlich, denn dieser Roman kommt einem zunächst sehr fremd vor. Man muß sich mühen, einen Zugang zu finden. Die Hauptfigur mit Namen Rausak, auf die alle Aufzeichnungen dieses Romans zurückgehen, hat ein anderes Verständnis von Erzählen, als wir es gewohnt sind. Rausak versteht Erzählen: „in Tonfall und Sinn wie errechnen, ermessen, erarbeiten, erwandern, erklettern, ertrotzen, Faktum an Faktum, schlichten, in großer Zahl so aufgelistet, daß Gesetze erkennbar werden müßten, wenn in der Wirklichkeit Gesetze herrschten“ (202).

Erzählen ist für Rausak der Versuch, die Wirklichkeit beschreibend zu bewältigen, obwohl sie so unübersehbar und komplex geworden ist, daß man sie eben nicht mehr beschreiben kann. „Nur zählen, mühsam heranzählen, vermeintlich immer näherzählen, stets von Fluten wieder abgetrieben, neu durchzählen, aneinanderreihen, außen abtasten, Rückgriff auf glaubhafte Sinneswahrnehmung ... Stotterexistenz ... ich bin, der ich bin, unqualifiziert, nicht genug erzählt, nichts erzählt" (202f).

Erzählen ist also nicht das Erdenken einer Geschichte, Erschaffung einer neuen Welt aus der Phantasie des Erzählers, Erzählen ist vielmehr ein notwendiger Versuch der Wirklichkeitsbewältigung, ein Lebensvorgang.

Man fühlt sich hier an den Roman von Max Frisch: „Mein Name sei Gantenbein" (Frankfurt 1964) erinnert. Das Thema dieses Romans lautet: „Ein Mann hat eine Erfahrung gemacht, jetzt sucht er die Geschichte seiner Erfahrung ..." (9). Max Frisch besinnt sich darauf, daß ein Mensch auf das, was ihm zustößt, ganz unterschiedlich reagieren kann; er kann das, was er erfährt, auf verschiedene Weise deuten und bearbeiten. Die jeweilige Bearbeitung und Deutung nennt Max Frisch "die zu suchende Geschichte der Erfahrung“.

Man kann so oder anders reagieren, man kann eine bestimmte Erfahrung so oder anders deuten, man muß eben eine Geschichte zu einer Erfahrung suchen. Unterlassen kann man das nicht; denn sobald jemand sich eine

Dr. Hermann Josef Spital ist Bischof von Trier und Vorsitzender der Publizistischen Kommission der Deutschen Bischofskonferenz. Der vorliegende Text geht zurück auf einen Vortrag auf der Hauptversammlung des Verbandes Katholischer Verleger am 29. Mai 1992 im Kloster Banz, Stuttgart. 
Erfahrung zu Bewußtsein bringt, hat er sie bereits in eine Geschichte eingekleidet. Erfahrung im Rohzustand kann nicht zu Bewußtsein kommen und damit auch nicht dem Menschen zu eigen werden.

Max Frisch sucht durch das Geschichten-Erzählen sich selbst und sein Leben zu verstehen; Rausak er-zählt, um die Wirklichkeit, in die er sich gestellt sieht, vor die Augen zu bekommen, ohne etwas auszulassen, um seiner Verantwortung für seine Kinder gerecht zu werden.

"Tatsächlich interessiert mich nichts als gefundene Wahrheit zugunsten der Kinder ... Ich möchte ohne Augenzwinkern einige Meldungen, Mahnungen, Ermutigungen verfassen. Ich schaue täglich in ihre Augen“ (16).

Rausak will nicht Irrgärten fabrizieren, die nur Verwirrung stiften; er will Hilfen geben.

Der Verfasser, der unter einem Pseudonym schreibt, ist nicht Schriftsteller von Beruf; er muß nicht um des Gelderwerbs willen schreiben. Er konnte (und mußte wohl auch) sich für dieses Buch 20 Jahre Zeit nehmen. Er ist Technischer Abteilungsdirektor in der Großindustrie, Computer-Fachmann, Lehrbeauftragter der Wirtschaftsuniversität Wien. In einer kurzen Vorbemerkung zu seinem Buch schreibt er: „Alle Personen, Handlungen und Ortsbeziehungen sind literarische Entwürfe; das umrissene Gebilde der „EBC“ entspricht keinem real bestehenden Unternehmen. Keine einzige aller im Text enthaltenen ,Unterredungen' wurde (leider) je wirklich geführt."

Matthias Mander führt die Erzählgestalt Rausak als jemanden ein, der das, was berichtet wird, aus Notizen „in Nächten, Eisenbahnwagen, Hotels, bei Unterredungen, zwischen Vortragsnotizen, an Stephanitagen, Pfingstmontagen, jeweils letzten Urlaubsstunden, auf Packpapieren, Kuverts, Buchrändern" (406) niedergeschrieben hat; insofern hat das Buch eine lange Entstehungsgeschichte und ist Stück für Stück zusammengewachsen. Wenn man es liest, kann man den Eindruck gewinnen, daß hier jemand seinen Zettelkasten mit einer Fülle von Gedanken, Einfällen, Erlebnissen veröffentlicht hat. Vieles erscheint fremd, zwingt zum Nachdenken; fast auf jeder Seite muß man innehalten, ein zweites Mal lesen, um den Gedanken zu verstehen. Mich fasziniert dieses Buch, weil ich mich in meinem Wirklichkeitsverständnis nirgends sonst so wiedergefunden habe wie in diesem Roman. In Rausak hat Matthias Mander eine Gestalt geschaffen, die in unserer modernen Wirklichkeit lebt, und das heißt: Nicht aus der Wirklichkeit isolierende Selbstbespiegelung wird geboten, sondern verantwortete Auseinandersetzung. Auch darin unterscheidet sich dieses Buch von vielem, was in der zeitgenössischen Literatur gängig ist. Die Wirklichkeit dieser Welt wird nicht als etwas dargestellt, das nichts anderes ist als „Material“ menschlichen Machens. Sie wird verstanden als etwas, das aufgegeben ist und darum ständig zu verantwortlichem Umgang herausfordert. Letztlich ist sie der Raum unserer umfassenden Gottesbeziehung, die nicht auf persönliche Erfahrungen eingeengt, sondern als etwas mit dem ganzen Leben zu Realisierendes verstanden wird. Rausak zitiert: „Die wörtliche Bedeutung des Ausdrucks ,Gott': das Gegebe- 
ne, das Vorgegebene, Aufgetragene, Verbindliche; unumgänglich, unübergehbar ..." (30).

Niemand, der nicht die ganze Wirklichkeit der Welt und seines Lebens akzeptiert und zu bestehen sucht, findet Gott. Man kann das Buch geradezu als ein „spirituelles Buch" bezeichnen, obwohl in ihm selten unmittelbar von Religion und Glaube gesprochen wird. Vielmehr prägt die Haltung zur Wirklichkeit den spirituellen Charakter fast jeder Seite.

Dem entspricht die Einstellung zur Politik. Einem jungen promovierten Akademiker, der ihm vorwirft, daß seine Ablehnung der weltlich gängigen Politik lediglich wehleidig sei, hält Rausak entgegen: „Durchschaust du die Eigensuchts-Mechanismen, den Menschenfraß denn nicht? Zyniker sagen, man müsse politisieren, weil man sonst selbst unter die Räder der Politik käme: Das ist es ja eben! Warum eigentlich? Deine engstirnige Besessenheit ist nicht verbindlich: Wahrscheinlichkeitstabellen sollte man aufrichten wie Evangelienbücher mit Kerzen und Weihrauch ... In jeder Geschichtsphase ist euch etwas eingefallen, auf Leben und Tod gegeneinander zu kämpfen, Ursachen nie angemessen, immer vorgetäuscht! Geplante Mißverständnisse, vergeudete Begabungen; nach schwieriger Ausbildung werden aus den Besten Parteisekretäre, Hauptpolemiker - andere Spitzenfunktionen hat die Welt nicht zu vergeben. So schaut sie denn auch aus: offene Hölle! Weil ihr, auch du, Thomas, an eine verbindliche, außerhalb von uns wirksame, ohne Schäden nicht zu verbiegende Wahrheit nicht glaubt. Deren Ratschläge hießen nämlich: Nachdenken, Reden lernen, Frieden halten, Arbeiten“ (265).

Freilich ergibt sich aus dieser Wirklichkeitssicht ein wenig auch die Verständnisschwierigkeit: Man kann dieses Buch nicht im Lehnstuhl lesen, um sich verzaubern zu lassen. Man muß sich vielmehr in den Lebensprozeß, der beschrieben wird, hineinziehen lassen; man muß sich in ein Gespräch begeben, selber Stellung nehmen, stets sich fragen, ob man das Berichtete auch so verstehen kann, wie es Rausak tut, oder nicht. Es gibt nicht einen überwölbenden Bauplan, der jeder Einzelheit sozusagen seinen Platz zuweisen würde und von dem her man in Ablehnung oder Zustimmung Stellung nehmen könnte; das Buch ist vielfältig, oft verwirrend, manchmal fast widersprüchlich: wie das Leben auch. Denn auch im Leben lernen wir nicht so sehr dadurch, daß wir eine Realität rational analysieren, um sie dann „abzuhaken“; vielmehr müssen wir uns auf einen im Grunde unabschließbaren Prozeß der Beziehung einlassen, in welchem sich nach und nach Wirklichkeit erschließt - wenn wir den Mut und die Geduld haben, es mit ihr auszuhalten. Zu einer solchen Haltung zwingt uns dieses Buch. Dabei wird gerade nicht behauptet, daß irgend jemand "die" Wirklichkeit objektiv erreichen oder beschreiben könnte. Vielmehr bleiben wir Menschen auf das Miteinander des Lebens und Sprechens angewiesen, um einen möglichst hohen - oder besser: ,verantworteten“ - Grad der Annäherung an die „verbindliche, außerhalb von uns wirkende Wahrheit" zu erreichen.

2. Um den Aufbau des Buches verständlich zu machen, möchte ich von dem schon genannten Anliegen des Herrn Rausak ausgehen. Er will seinen Kin- 
dern zuverlässige Orientierungshilfen geben, weil er die Verantwortung vor seinen Kindern spürt: „Ich schaue täglich in ihre Augen." Aber wie sollen die Kinder sich orientieren in einer Welt und in einer Gesellschaft, die unübersichtlich und unverständlich geworden ist? Es gibt keine Wertmaßstäbe mehr, die die Gesellschaft strukturieren, die man den Kindern, mit unterstützenden Hilfen vielleicht, yermitteln und als Orientierung mitgeben könnte. Das christliche Abendland ist endgültig vergangen. Es ist nicht nur als Gestalt vergangen; es ist vielmehr auch als Angebot einer Weltsicht vergangen. Eine Summa Theologica, die von einer in der Gesellschaft mehr oder weniger gemeinsamen philosophischen Weltsicht ausgeht, ist prinzipiell nicht mehr möglich. Unsere Welt hat alle Systeme gesprengt und begegnet uns in einer unüberschaubaren Vielfalt. Was soll also ein Vater seinen Kindern vermitteln, wenn sich keine in sich stimmige und darum weiterzuvermittelnde und einsichtig zu machende Weltanschauung (im Sinn einer begriffenen Übersicht) mehr anbietet?

Diese Frage treibt Rausak um, quält ihn. Er kann nur seine persönlichen Erfahrungen weitergeben, was sonst? Aber sind diese Erfahrungen verbindlich, wo sie doch nur seine persönlichen Erfahrungen, und nicht etwa objektiv gültige Tatbestandsbeschreibungen sind? Und wie formuliert man sie, damit sie so verstanden werden, wie sie gemeint sind?

"Unablässige, inständige Suche nach einem Mitteilungsmodell, Benachrichtigungssystem, das Einsichten transportiert, die teils lebensbegründend, wenngleich unaussprechlich sind, teils erst während der Verladeversuche entstehen ..." (201).

In früheren Zeiten wurden die von den Eltern an die Kinder übermittelten Wertmaßstäbe dadurch plausibel, daß sie auch in der Gesellschaft galten, in die die Kinder hineinwuchsen. Angesichts der immer rascher fortschreitenden Entwicklung ist diese Kontinuität heute zerfallen. Wir leben in einer prinzipiell pluralistischen Gesellschaft: Und das ist nicht mehr rückgängig zu machen. In dieser Situation geht es für den verantwortungsbewußten Vater um ein Doppeltes: Zunächst muß er jemand werden, der verbindliche Erfahrungen zu machen imstande ist; sodann muß er eine Form finden, diese Erfahrungen den Kindern zu übermitteln.

Aber wie macht jemand verbindliche Erfahrungen? Offensichtlich muß er dazu zunächst einmal sein Leben ernst nehmen. Von diesem Ernst gegenüber der Wirklichkeit und dem Leben ist jede Seite des Buches geprägt. Das macht den Leser betroffen und spricht ihn unmittelbar an.

Nun ist aber - wie schon gesagt - unsere Welt so unüberschaubar geworden, daß man ihre Wirklichkeitsfülle in kein System mehr bringen kann. Was kann man tun, wenn man gleichwohl die Wirklichkeit verantwortlich annehmen und ernstnehmen will? Man kann sie wenigstens auflisten, sammeln, registrieren. Einfach nebeneinander aufschreiben, ohne etwas auszulassen. Das erste Buch des Romans enthält darum „Listen“. Rausak schreibt, daß diese Listen Ähnlichkeit haben mit dem Wachstum der Mineralien: Minerali- 
en wachsen ja nicht wie Lebewesen nach einem organischen Bauprinzip, vielmehr gehen sie „nur äußerliche Verbindungen ein, fügen Atom zu Atom, ohne Mittelpunkt. Kristallstruktur, geometrisches Netz“ (10).

Die einzelnen Erfahrungen werden also nicht einem vorgegebenen System eingefügt und dadurch zueinander in wertende Beziehung gebracht. "Das System entspräche nicht der Erfahrung, wirkte klebrig, als Fesselung" (10). Rausak bestreitet nicht, daß die von uns erfahrenen Wirklichkeiten Teil eines umfassenden Ganzen sind; aber der Bauplan, das "System“ dieses Ganzen ist unserer Kenntnis entzogen. Wir können es aus den uns bekannten Ausschnitten nicht konstruieren: „Hierfür reichen die Indizien nicht aus ...“ (10). Zwar spürt auch Rausak, wie jeder Mensch, das Verlangen nach einer systematischen Zusammenschau, nach Übersicht und Ordnung, weil eine solche Zusammenschau die Orientierung erleichtert. Er versagt sich diese Zusammenschau, weil er ehrlich ist gegenüber der Wirklichkeit, wie wir sie heute erfahren. Er läßt sich auf unsere Welt ein, ohne zu verkürzen; er verzichtet auf jede vereinfachende Ideologie oder Utopie.

Er empfindet das als schmerzhaftes Paradox: „Während seine ganze Existenz auf vertikale Bezogenheit gespannt ist“, also auf ein Du, das Du anderer Menschen und letztlich das Du Gottes: „Während also seine ganze Existenz auf vertikale Bezogenheit gespannt ist, keine Minute wach oder schlafend vergeht, ohne daß er Tiefbohrungen vorantriebe, bewegt er sich, handelt er nur horizontal, vertraut nur Plattem, Unmittelbarem. Erkennbar ist ihm nur die Ebene" (13).

Unser Leben besteht aus Alltäglichkeiten. Und doch wissen wir, daß unser Leben nicht etwas Alltägliches, sondern etwas Einmaliges und zu Verantwortendes ist. Aber die Maßstäbe für diese Verantwortung kann Rausak nicht aus der alltäglich ihm begegnenden und unüberschaubar gewordenen Welt gewinnen: „Es müßte eine unvorstellbar große Systemkugel sein, in deren Basispunkt er lebt, der bis an den Horizont reicht und noch keine Krümmung erkennen läßt“ (13). - Diese Maßstäbe findet er darum niemals in einem noch so intelligenten Kalkül, sondern allein im personalen Gewissen.

Rausak sieht also keine andere Möglichkeit, als die ihm begegneten Wirklichkeiten aufzulisten. Aber aufgelistete Wirklichkeit gibt noch keine Erfahrung. Mit den Listen allein können die Kinder nichts anfangen. Listen können vielleicht interessant sein, aber sie bleiben unverbindlich, solange der Bezugspunkt fehlt. Wenn nun der Bezugspunkt nicht aus einem System gewonnen werden kann, aus allgemeinverbindlichen Erkenntnissen, welche sich aus den Erfahrungen sozusagen herausfiltern, was kann es dann für einen Bezugspunkt geben? Dieser Bezugspunkt ist die Person dessen, der da seine Wirklichkeitsbegegnungen niederschreibt. Erst in Beziehung zu dieser Person, vorausgesetzt, daß sie den Kindern etwas bedeutet, werden die Listen interessant. Weil Rausak seinen Kindern etwas bedeuten will, versucht er, als Mensch und als Christ überzeugend zu leben.

Überzeugend ist, wer ehrlich ist. Darum fügt Rausak dem Buch der „Listen“ ein Buch der „Klagen“ hinzu: „Rausaks Klagen sind ... Koten, zugleich 
Sextant, Meßinstrument, um den Klagenden zu orten, zu kennzeichnen, festzulegen, Selbstberingung, Wasserwaage zur Vermessung einer Lebensbasis: Gänzliche Offenlegung, ehe Briefe verfaßt werden, um Einordnung, auch Zurückweisung seiner Ratschläge zu ermöglichen. Daß die Kinder sich von ihm abstoßen oder auf ihn bauen könnten, aus Ruinen brauchbares Gestein für neue Bauten gewännen“ (199).

Die Selbstbeanspruchung, der sich Rausak unterzieht, um seinen Kindern etwas Verläßliches mitzugeben, wird an dieser Stelle deutlich. Sie macht, das sei noch einmal gesagt, den Leser betroffen und fesselt ihn immer wieder, trotz der anspruchsvollen Lektüre.

"Nicht täuschen, nicht eitel sein, nur der Aufgabe entsprechen, euch zu raten, euch für Schmerzen, Irrungen, Versuchungen zu stärken. Ich bin am Leid mitschuldig, das ihr erbt" (199).

Wer sich so seiner Lebensaufgabe stellt, wissend um sein Versagen, sein unausweichliches Eingebundensein auch in die Schuld einer Zeit und Welt, ohne sich stolz zu distanzieren, aber mit immer wieder neu aufgenommener Bemühung, das Beste zu tun: Der hat einige Aussicht, vielleicht doch überzeugend zu sein für seine Kinder. Das ist die Voraussetzung für die Wirksamkeit der Lebenshilfe, die Rausak seinen Kindern geben möchte.

"Drei Stufen dieser in stets neuen Anläufen versuchten Hilfe: Listen zeichnen die Umwelt, Klagen geben mich zu erkennen, Briefe beraten euch" (199). Den Büchern der "Listen" und der „Klagen" wird also noch ein drittes Buch der "Briefe“ an die Kinder beigefügt. Die "Briefe" enthalten Warnungen, Zurufe, Gewißheiten, Ratschläge.

Nun lebt der Mensch ja nicht nur in seiner Familie. Er muß sich in Arbeit und Beruf bewähren. Auch davon ist die Rede. Ein mit „Vorberichte" überschriebenes Buch kennzeichnet jugendliche Grunderfahrungen Rausaks, die zu seiner Persönlichkeitsbildung beigetragen haben. Dann folgt ein weiteres Buch mit „Berichten“: In ihm sind die beruflichen Begegnungen und Erfahrungen zusammengetragen. Ein Buch mit dem Titel „Preisungen“ berichtet über das, was Rausak als ermutigend und heilend erfahren hat; ein letztes Buch mit dem Titel „Stufen“ beschreibt die für dieses konkrete Menschenleben wegleitend und in gewissem Sinn abschließend gewordenen Begebnisse. Mander schreibt diesen drei Schlußkapiteln in der kurzen Vorrede zu seinem Buch „kathartische“, also reinigende Funktion zu im Sinn einer abhaltenden Prophetie. Wir sollen sozusagen die Summe dieses Lebens anschauen und versuchen, es besser zu machen.

3. Nachdem ich nun den Aufbau des Buches erläutert habe, muß ich noch ein paar Bemerkungen zur Erzählform machen. Aus den Zitaten ist Ihnen vielleicht schon aufgefallen, daß die Abschnitte teils in der Ich-Form gefaßt sind, teils aber auch in der dritten Person erzählt werden, wobei Rausak sich selbst bei seinem Namen nennt. Im Wechsel dieser Erzählformen geht es so sehr hin und her, daß nicht leicht eine Systematik darin zu erkennen ist. 
Wenn ich mich nicht täusche, weist der Verfasser durch diesen Stilwechsel darauf hin, daß wir das, was uns im Leben begegnet, in höchst unterschiedlicher Intensität verarbeiten. Manches wird einfach zur Kenntnis genommen und registriert; anderes geht uns unter die Haut, wir machen es uns zu eigen, wir können darüber reden als von etwas, das uns zur Überzeugung geworden ist. Von seiner Überzeugung kann man nur persönlich sprechen; was lediglich registriert ist, vermittle ich in der dritten Person. Der Mensch kann sich selbst zum Gegenstand seiner Betrachtung und seines Nachdenkens machen.

Schließlich möchte ich noch mit einem Wort auf den Stil eingehen. Sie haben aus den Zitaten wohl auch schon entnommen, daß Rausak in einer Art Telegrammstil schreibt. Dabei häuft er für jede Wirklichkeit, die er benennen will, die Ausdrücke. Er tastet das Gemeinte gleichsam rundherum ab; er versucht, es einzukreisen, weist durch jeder der gehäuften Ausdrücke in anderer Weise auf es hin. Auch in Sprache und Stil wird somit erkennbar, daß Rausak sich klar darüber ist, die Wirklichkeit durch sein Er-Zählen nicht präzise zur Sprache bringen zu können. Dabei spiegelt diese Sprachform die für unsere Wahrnehmung ungeordnete, zufällig erscheinende Wirklichkeit wider, die sich eben nicht auf einen gemeinsamen Nenner bringen und überschaubar machen läßt. Sprache hat Verweisungscharakter - sie ist und kann nicht fotografische Abbildung von Wirklichkeit sein. Sprache ist immer auf das Gespräch aus, richtet sich an ein $\mathrm{Du}$; durch die Häufung der beschreibenden Worte werden wir in ein Gespräch hineingezogen; wir müssen uns fragen, welchen dieser Ausdrücke wir in dem betreffenden Zusammenhang für den genauesten halten. Dieser Stil ist eine Zumutung im ursprünglichen Sinn des Wortes. Man kann dieses Buch nicht passiv in sich aufnehmen; man muß sich in ein Gespräch begeben.

Schließlich muß hier noch die ganz außergewöhnliche Fülle von Bildern und Metaphern genannt werden, die unser Buch bietet. Bilder laden zum Verweilen ein; man muß sie betrachten. Sie sagen mehr als eine „objektive“ Beschreibung; sie sprechen eine Sprache, die den Partner nicht in eine lediglich annehmende, festgelegte Rolle zwingt, sondern ihn zum eigenen aktiven Aufnehmen einlädt. Auch das ist eine hohe Anforderung, sollte und kann aber zugleich auch ein verlockender Anreiz sein.

II.

1. In einem zweiten Teil will ich versuchen, in das Buch dadurch einzuführen, daß ich einzelne Abschnitte erläuternd vorstelle.

Im Vorbericht 1 mit dem Titel „Die Hatz" beschreibt Rausak, wie sie als Jungen auf dem Nachhauseweg von der Schule ein sie innerlich ganz in Anspruch nehmendes Spiel veranstalteten. An einer Brücke „zerrten wir unsere Ranzen vom Rücken, rissen Blätter aus den Heften, weiße, unbeschriebene Seiten, die schwerfällig neben dem Brückenkiosk ins Wasser flatterten" (98). Auf diese nun wie Schiffe im Fluß treibenden Heftseiten 
warfen die Jungen Steine, um sie zu versenken. Das gelang nicht; auch wenn sie die Blätter trafen, rutschte der Stein ab, und das Blatt kam wieder an die Oberfläche. Rausak erzählt: „Unsere Waffen verbrauchten sich. Ein Stachel schnitt in unseren kindlichen Willen, der so ohnmächtig war, beschämend vor dem Flüchtigen, Widerstandslosen, das - kaum unseren Händen entflattert - unnahbar wurde, jenseitig, voll langsamer Würde, die selbst unsere wildesten Treffer kaum störten" (100).

Als dann die Heftseiten die letzte Brücke passiert hatten, mußten die Jungen aufgeben. „Wir redeten nichts. Die Hatz kochte noch in unseren Körpern. Aber sie begann sich in diesem Augenblick vollendeter Demütigung in ein Glücksgefühl aufzulösen. Weiter schweigend, die Hände an den verschwitzten Stirnen, schauten wir gegen die Sonne auf das Fortfließen unserer fröhlichen Inseln. Wir konnten sie im Glitzern noch sehen, bis sie weit unten, gerettet, durchs Fluder der Rösselmühle in das zu uns herüberrauschende Geheimnis eindrangen" (101).

Die Szene beschreibt die Zwiespältigkeit menschlichen Verhaltens gegenüber der Wirklichkeit dieser Welt. Der Mensch will diese Wirklichkeit beherrschen, er will sie in seine Gewalt bekommen, selbst, wenn er sie zerstört; die Wirklichkeit aber erweist sich letztlich als stärker: Und das wiederum gibt dem Menschen eine Geborgenheit, gibt ihm Lebensraum und Boden unter die Füße. Die Demütigung des Mißlingens wird zum Glücksgefühl unzerstörbarer, bergender Wirklichkeit. Wo der Mensch die Wirklichkeit so anzunehmen lernt, kann er für sie danken.

2. Der Vorbericht 'zwei' trägt die Überschrift „Das Mißlingen“. Er beschreibt ein anderes Jugenderlebnis der Hauptperson. Oft hat Rausak sich an Sommerabenden nach dem Schlafengehen heimlich wieder aus dem Zimmer geschlichen und ist in die Scheune gekrochen, wo die Fledermäuse flogen. Vorher hatte er Falläpfel gesammelt: Nun versuchte er, mit den Äpfeln, die ihm so geheimnisvoll erscheinenden Fledermäuse zu treffen, um eine von ihnen fangen zu können. Trotz aller Mühe und Anstrengung gelang das nicht. Er mußte sich geschlagen geben.

"Aber immer noch flogen die pelzigen Sendboten des ebenso Geheimen wie Vertrauten, ja in tiefster Seele erschütternd Verwandten, Verbindlichen nekkisch unbeholfen in Leibeslänge um mich. Ihr Quietschen mußte aus Gesichtern kommen, deren Anblick wohl einer Botschaft aus dem Jenseitigen gleichkam. Jetzt, da ich körperlich geschlagen war und erschöpft in meiner Mulde lag, war mein Verlangen eher noch inniger und dringender" (105).

"Oft konnte ich die großen Ohren steil ins Dämmern ragen sehen. Und spitzig schlangen sich ihre Zurufe durch die vibrierende Luft. Meine Äpfel schwirtten jetzt seltener, aber gezielter gegen ihre Leiber, stießen auch - wenn ich gut warf - derb und krachend gegen sie, so daß ich meinte, sie erlegt zu haben, wenn sie mit der Frucht zusammen flatternd abstürzten: Aber immer war es nur ein kurzer Ringkampf, der sich knapp über der Erde löste und die Fledermaus ins Höhere entließ. Dann suchte ich den Apfel“ (106). 
"Im fahlen Licht erschienen mir Büsche und Zäune riesengroß, und der Klimmzug zur Kammer überforderte mich fast. Im Bett erst betastete ich atemlos die heimgetragenen Äpfel nach Kerben, von denen ich meinte, sie stammten aus den Krallen und Zähnen der Fledermäuse, die mit ihnen in der Luft gekämpft hatten" (106).

Ich verstehe diesen Erfahrungsbericht als ein Bild für den Versuch des Menschen, hinter das Geheimnis der Wirklichkeit zu kommen. Er spürt dieses Geheimnis; es „umflattert ihn“ ununterbrochen; aber greifen kann er es nicht. Allenfalls findet er Spuren dieses Geheimnisses in seinem Leben.

3. Ich wähle aus dem Buch der „Vorberichte“ - der Kindheitserfahrungen - den dritten mit der Überschrift: „Die Ankunft“. Er berichtet uns von dem Überschritt des Knaben, der auch für alles offen, sozusagen „ungebunden“ ist, in die einmalige und ganz persönliche menschliche Bindung. Rausak hat seine Mutter in früher Kindheit verloren. Als Vierzehn- oder Fünfzehnjähriger fährt er mit dem Fahrrad über die Grenze nach Deutschland zu dem Friedhof, auf dem seine Mutter begraben liegt. Er mußte im Münchener Kolpinghaus übernachten. Davon berichtet er: „Ich wachte und hatte Zeit, die Befürchtung zu überlegen, die mich schon tagelang drückte: Ich würde am Grab nicht weinen können! Ich hatte sie ja nie gekannt" (108).

Auch auf der Fahrt am nächsten Tag, als er dem Friedhof näher und näher kommt, werden die Ängste um sein Verhalten vor dem Grab wieder wach. Er schreibt: „Eine noch tiefere Härte lag jetzt in mir. Ich fürchtete, durch sie meine Tote zu beleidigen, ja, ihr sogar zu schaden. Lieber wollte ich nicht beten, als schmerzlos etwas hersagen. Aber am Grab der Mutter, vor das man zum ersten Mal tritt, nicht beten?" (109)

Als er dann schließlich gegen Abend am Grab stand, bricht das Herz auf: „Ich weinte laut auf; ich kniete betend in dieser Erde; hier war Endgültiges, Unheilbares geschehen für meine Mutter und für mich. Meine Augen werden offenbleiben, immer offen; ob ich dränge oder fliehe, das wird nie mehr gut, bis zuletzt nicht“ (109).

Die Einmaligkeit des Personseins und des jeweiligen Schicksals hat den jungen Rausak eingeholt.

4. Von den zwölf Kapiteln aus dem Buch „Berichte“ will ich nur eines hier vorstellen; der Bericht 4 ist überschrieben: „Die Flucht“. Rausak nimmt uns mit in ein Konzert, in das er aus Liebe zu seiner Frau Francine gegangen ist. Doch das Konzert wird für ihn überlagert durch eine gestörte Beziehung. Einer seiner Vorgesetzten, Führungsratsmitglied der Erz-Blech-Chemie, geht durch die Reihen und nimmt vorne Platz. „Mit diesem Mann verbindet Rausak jener Zweifel, der der Rest einer Verehrung ist, vor vielen Jahren, einem fernen Vorbild aufrichtig entgegengebracht. Die Gefühle sind jetzt kompliziert, voll Enttäuschung: Snanz verläßt demnächst die Erz-Blech-Chemie - er wird Professor in Innsbruck" (131). 
Nach einigen anderen Gedanken greift Rausak diesen Faden wieder auf: „Das Snanz-Thema heißt für mich: Wie gerecht darf man inmitten von Unrecht sein? Und wie gerecht ist das dann noch? Snanz hat viele angeleitet, die Wirtschaftswelt in Ordnung zu finden, sich im Beruf sicher zu fühlen; scheinbar redlich eingeschätzt und bewertet vom System, haben sie sich an Arbeit verausgabt, statt gekämpft, waren zufrieden, daß man ihnen stets freundliche Anerkennung zollte.

Aber hat Snanz nicht gewußt, daß er die Risiken nicht allein tragen darf, daß er uns anderen Recht auf Information gewähren, ja erkämpfen müßte? Rausaks Verdacht lautet, daß Snanz die reale Sachlage verschweigt, die unerhörten Rückschläge allein erträgt, sie nirgendwo deutlich kommentiert, daß er mangelnde Gegenmaßnahmen nicht laut und öffentlich fordert" (132).

Hier zeigt sich eine entscheidende Grundauffassung des Romans: Nur ein wirkliches Miteinander aller in der Industrie Tätigen würde die heute anstehenden Probleme lösen können; aber auch: Nur so etwas würde den Menschen als Menschen fordern und ihn damit in seinem Menschsein ernst nehmen. Rausak steht zur Großindustrie, weil es für das Überleben der vielen Menschen auf unserer Erde dazu keine Alternative gibt. Er sieht die unmenschlichen Strukturen unserer industriellen Großgebilde und kämpft um ihre Vermenschlichung. Er versagt sich aber utopischen und unerfüllbaren Forderungen. Sie würden ihm vielleicht bei manchen Menschen Beifall und Gefolgschaft einbringen, müßten sich aber über kurz oder lang als unrealisierbar erweisen und würden dann herbe Enttäuschungen hervorrufen. Derlei Geschrei erscheint ihm verantwortungslos und widerspricht zutiefst der Haltung eines in der Industrie tätigen Menschen. Denn dort geht man mit Realitäten um und kann sich keine Träume leisten. Rausak wehrt sich leidenschaftlich gegen diejenigen, die utopischen Idealen nachlaufen, weil er das als Flucht vor der gegebenen Wirklichkeit empfindet. Andererseits aber besteht er darauf, daß alle Mitarbeiter das Recht auf eine umfassende Information haben, weil das die Voraussetzung eines menschlichen Miteinanders erst schafft. Seiner Meinung nach würde das auch die gemeinsame Anstrengung, die in Krisenzeiten zur Erhaltung der Arbeitsstätten notwendig sein kann, erst ermöglichen.

Im folgenden wird die Atmosphäre der Musik beschrieben; Rausak empfindet das Schwärmen in den Tönen als Flucht vor der rauhen Wirklichkeit. Dann wird der Faden des Nachdenkens über den Weggang des Topmanagers wieder aufgegriffen: „Rausak sieht das Distanzierte, immer noch verehrte, einst fast geliebte Profil fünf Reihen vor sich, den schimmernden Violinen zugewandt. Die gesuchte“ Objektivität, mit der Snanz seinen Rücktritt betreibt, die wahren Gründe seines Ausscheidens verbirgt, seine einmalige Chance, einen Strahl Wahrheit auf die gefährliche Wirtschaftspolitik zu werfen, für sich und uns vergibt. Entzug zu akademischen Ehren hinauf. Glasur unzerbrochen erhalten. Der beste König sei der, der nicht regiert ... Rausak empfindet die Nähe dieser Tragödie als körperlichen Schmerz" (133).

In diesem Bericht scheint die Welt auf, in der Rausak seinem Beruf nachgeht. Er ist Technischer Abteilungsleiter in der ,Erz-Blech-Chemie'. In den ver- 
schiedensten Berichten wird die Problematik der Großindustrie, das oft unverantwortliche Handeln der Mächtigen, das Scheitern mancher, die sich bis zum Letzten engagiert haben, die Auseinandersetzung zwischen den technischen Fachleuten und den übergeordneten Managern und die Verflochtenheit mit der Politik beschrieben. Rausak setzt sich leidenschaftlich für das oft mißachtete Recht des Menschen in solchen Strukturen ein. Die Klarsichtigkeit, das Engagement für den Menschen, Kritik und Bewunderung ergreifen den Leser; es wird oft scharf beschrieben; deutlich abgelehnt, aber nirgendwo findet sich Zynismus. Auch das härteste Urteil ist von einer Sympathie für alles Menschliche getragen. Ein letzter Satz aus dem Bericht „Die Flucht": „Snanz setzt vielleicht auf Zeitgewinn, verschliffenen Übergang, Morbidität der Fronten, langfristiges Unterrosten, Hinterspülen der Macht. Diese Strategie erfordert freilich Opfer - aber nicht von ihm, er bedauert nur die Tragödie, die andere erleiden ... Diesem Mann habe ich vertraut, habe geglaubt, daß es um Sachprobleme so stünde, wie es seiner oft angedeuteten Sicht entspräche. Ohne eine ordnende Handlung zu setzen, übersiedelt nun Snanz nach Innsbruck, wird Professor. Zorn, auch Eifersucht, Zweifel an seiner eigenen Sicht befallen Rausak" (135).

5. Aus den neun Kapiteln des Buches der „Klagen“ greife ich die sechste Klage heraus mit der Überschrift „Ysop“. In diesem Abschnitt setzt sich Rausak mit den Gefahren, aber auch mit den Möglichkeiten der Literatur, also des Bücherschreibens, auseinander. Die Szene, in welche die Gedanken eingestreut sind, wird folgendermaßen beschrieben: „Von Halbenrain grazwärts hinter einem Viehtransporter: während aus dem Autoradio die Stimmen einer Diskussion zwischen witzelnden, übermütigen Kraftautoren und abschätzig behandelten, beleidigten Lehrern tönen, rinnt Schweinekot über die Bordwand auf die Hinterräder des Lastwagens, spritzt auf Rausaks Windschutzscheibe, verläuft stinkend auf dem Glas, verschmiert vom Scheibenwischer" (255).

Das ist eine Situation, wie viele von uns sie ganz alltäglich erleben, aber sie hat hier eine gleichnishafte Bedeutung - wie fast immer in diesem Buch. Rausak beschreibt, was ihm bei der im Autoradio gehörten Diskussion einfällt: „Erinnerung an Frau Watzinger von der Revision, Tierfreundin, auch fromm, kiloweise Polentafutter für Stadtvögel zwischen parkende Autos streuend, oft entschuldigend erwähnte Kindheit im Hinterhof einer Fleischhauerei: sie beklagte die Nachrichten solcher Schriftsteller als Essigschwämme, auf Ysop gegen dürstende Lippen gepreßt. Ihr beklemmendes Gefühl während Kulturnachrichten im Fernsehen, eigentümliche Autorenversammlung: erbarmungslose Herren, die außer einem autistischen, orgiastischen Stolz nichts antreibt, von denen außer Entblößung, kalter Sezierung, Verdammung, Verstoßung nichts zu erwarten ist ..." (255).

Und dann fällt Rausak ein Gespräch ein, das er vor einiger Zeit hatte: „Wuggenik allein im Postzimmer, vergangenen Jänner zu Rausak: Literatur lebe als Schmarotzerin an der Menschheit wie die Tochter von der Nadel im Leib ihres invaliden Vaters, den sie nicht operieren lasse, weil sie vom Schmerzensgeld profitiere. Er könne einen Roman schreiben, schreit Wugge- 
nik immer, aber er sei nicht der Schwindler: Ruderbootwettbewerbe in trockener Halle vor dem Spiegel, Strömungsmesser an künstlich gepeitschten Kanälen“ (258).

Dann fällt Rausak wiederum ein anderer Aspekt ein: „Dankbarkeit erfüllt ihn beim Gedanken an Tausende Arbeitsstunden hinter Büchern, Papieren, Notizen, Korrekturen, die er sich als Lektüre, als lebenzeugende, lebensrettende Leseschätze einverleiben durfte" (258).

Es ist schon spannend, was jemand, der selbst schreibt, über Literatur denkt. Rausak läßt die unterschiedlichsten Stimmen zu Wort kommen: „Tröster Froling, einziger Dichter der Erz-Blech-Chemie, drei Lyrik-Bändchen ... stiller, überfreundlicher junger Angestellter in weißem Mantel, spitze Bleistifte in der Brusttasche, alte Lochkarten winzig beschrieben. Er lächelt bei dem Wort Wirklichkeit: Was Maurer dem Leib, seien Dichter der Seele: bauen Höhlen, in denen erträgliche Bedingungen herrschen, Schutz vor Illogischem, Absurdem, Regellosem, schon der erste gefügte Stein, das erste Wort seien unwirklich. Sinnloses Geschehen einzuholen, einzufangen sei gar nicht die Absicht, sondern es abzuhalten, auszusperren, abzudrängen, zu filtern, in Ordnung zu verwandeln, zu rhythmisieren, reimen, klären, auch verklären, das hieße Menschen retten" (259). Das wäre also Literatur als Zufluchtsort vor der harten und in ihrem Sinn uns nicht ohne weiteres erschlossenen Wirklichkeit.

Wieder eine andere Ansicht: „Schärfster Feind mancher Literaturerscheinung ist Verkaufsdisponent, Beethovenpianist, Firmendenker Zwigott ... wie einige Artisten von der durch die Geschichtslawine eingedrückten Wand abrükken, gegen die wir mit Stirnen und Fäusten gestemmt stehen müssen, schweißgebadet in brechende Bohlen gespannt, Eisflut, Schnee- und Felsdruck abzuhalten: und wie sie in sicherer Ecke dieses Raumes schmusen, Frisuren ausprobieren, Barttrachten züchten. Überzogener Klassenkampf, Sexualierung, Aleatorik: Frechheit, mit der sie Handlungen geschehen lassen im Tonfall amerikanischer Kurzkrimis: brutaler Unernst, ironische Grausamkeit, Parodie statt Salvierung. Weil ihr Kopf für keine Konzeption ausreiche, müsse nicht der Kopf geschult, sondern die Welt zerstört werden. Sinn sei nicht machbar, aber Unsinn sei machbar. Utopische Orientierung der Kunst diene grundsatzloser Politik" (259f).

Lassen Sie mich darauf aufmerksam machen: Hier findet eine gedrängte Auseinandersetzung mit dem Phänomen des Literaturschreibens statt: aber nicht in theoretisierender Form: Jede Auffassung verkörpert sich in einer Person, einem bestimmten, konkreten Menschen, der Rausak begegnet ist. Das ist programmatisch: Nicht systematisierende Theorie mit argumentativem Hin und Her, sondern allein der Mensch gilt.

Auch möchte ich hier aufmerksam machen auf das packende Bild der Situation. Wir stehen vor der „durch die Geschichtslawine eingedrückten Wand, gegen die wir mit Stirnen und Fäusten gestemmt stehen müssen, schweißgebadet in brechende Bohlen gespannt ..." - so empfindet Rausak 
das Stehen in der heutigen Welt, die, soll sie uns Menschen nicht vollends aus den Fingern gleiten, den Einsatz aller erfordert, gerade all derer, denen es um den Menschen zu tun ist. Aussteigen ist leicht; sei es ein Aussteigen in billige Literatur oder sei es ein Aussteigen in eine Drogenwelt. Aber wer aussteigt, fehlt bei der uns allen gemeinsam aufgegebenen Weltbewältigung.

Wie so oft denkt Rausak auch in diesem Zusammenhang an seine Kinder immer noch auf der Fahrt hinter dem mit Schweinen beladenen Lastwagen. Ihm fällt ein: „Wie unsere kleine, fünfjährige Anna vom sechsjährigen Max und der vierjährigen Klara im Vorzimmer umarmt wurde, als sie zur Operation mit mir ins Spital ging. Ihr kleines, grünes Hubertusmäntelchen, ihre blaue Zipfelmütze, ihre Augengläser und dahinter das brave Gesichtchen. Francine und ich mußten uns abwenden ... Dann sitzt da irgendwo in schmierigen Hinterhöfen und Kellerbuden eine besoffene Clique beisammen: diese Kinder sollen von lieblosen Augen gemessen, von geilen Fingern betappt werden, weltweite Publizität des Miesen ermutigt Abirrende, gegen die ich wehrlos stehe; jetzt noch die Köpfe der Kinder gegen meinen Schoß gedrückt, aber wie lange kann ich sie noch schützen?" (260)

Dann zum Schlưß seine eigene Einstellung: „Rausak nimmt zwar keine Nähe zur Kunst ein. Aber die jahrelangen Aufzeichnungen für seine Kinder ergeben äußerliche Parallelen: Arbeit, die er letztlich nur für sie leistet, auch wenn er die Papiere nie übergäbe - er hat ihnen damit einen Vater mit verläßlicher, Jahrzehnte überbrückender Berufserfüllung erhalten“ (262). Das heißt also: Das Schreiben stabilisiert ihn auch selbst, weil er sich so mit der Wirklichkeit auseinandersetzt.

6. Aus dem drei Kapitel umfassenden Buch der „Preisungen“ stelle ich die Preisung 1 vor mit der Überschrift: „Anrufe“. Rausak beschreibt Szenen, in der Menschen anderen Menschen ganz selbstverständlich geholfen haben. Glückliche Begegnungen, so wird eine genannt: „Im Schnellbahnwaggon um 6.0o Uhr früh den pensionierten Schleifmeister Pribor nach zwei Jahren wiedergetroffen, herzlich begrüßt: so früh auf, hübsch gekleidet; er lächelt verlegen; Liebesgeschichte in Puchberg! Rausak drückt ihm voll Freude fest den Arm: Gratuliere Ihnen! Mein Gott, was haben Sie in all diesen Jahrzehnten geleistet! Perlen rinnen ihm aus den alten, einsamen Augen" (298).

Und dann eine Charakteristik: „Friedensstifter - ohne es zu wissen! Die ihre Arbeit gar nicht anders ansetzen als in selbstverständlicher, gestaltender Annahme des Bestehenden. Unter Absehung von sich selbst leben. Einzig Festes in rasend abkippenden Jahren: Jene Sekunden, in denen einander zwei Blicke verstanden" (298).

Rausak denkt an so viele, denen er begegnet ist, und schreibt wieder: „Die guten Menschen in der Fabrik, ohne egozentrische Perspektive, entscheiden im Zweifel immer für die Gegebenheit und ihre Regeln, nicht für eigene Vorteile und Schwächen: Einzige nachhaltige Weltveränderer - Verwandler von Materie in Humanes" (299). 
In den Preisungen wird auch etwas von der emotionalen Empfindung Rausaks greifbar: „Liebesüberfülle beim Anblick der in Spätwintersonne vor dem IBMHaus auf die Straßenbahn wartenden Menschen. Ihre völlige Hilf- und Interesselosigkeit. Mit ungeheurer, unbedankter Anstrengung das Leben fristen, dem nichts abzugewinnen ist; ausgeliefert ziellosen Regierungen, pragmatischen Parlamenten, vernünftigerweise jederzeit der Vorzug für das Nicht-Sein, und doch durchhalten, versteinerte Fragen in der Brust ... Rausaks Dankbarkeit für das Durchhalten dieser Menschen! Schweigender Gottesbeweis. Preisung aus zerfurchter Stadt. Immer an diesem Ort: Schwedenplatz ... die Assoziation zum verstorbenen Präsidenten Gstötterbauer. Seine Zentralaussage: Wüßten alle Menschen von der noch zu leistenden Arbeit, der unterschiedlichen Fristigkeit ihrer Entscheidungen, von den geringen Opfern, die sie bringen müßten für klar erkennbare Besserungen, sie wären selbstlos, wohltätig, begeistert. Doch wurde von den Machthabern schuldhaft kein Informationssystem entwickelt, das die Aufgaben übersichtlich gemacht hätte - aber nur in solcher Rationalität könnte diese Epoche ihre Ethik begründen“ (301f).

Rausak glaubt an den Menschen, vor allem an den einfachen Arbeiter. Er mißtraut ein wenig den Funktionären, welche es freilich auch geben muß. Wie wäre es möglich, beide Gruppen und Tätigkeitsfelder zueinanderzubringen? Wie ließe sich ein Miteinander aller Menschen an der gemeinsam gestellten Aufgabe ermöglichen: Das ist eine Frage, die immer wieder zwischen den Zeilen zu lesen ist.

7. Aus dem Buch der sieben „Briefe“ greife ich den Brief 5 heraus, der überschrieben ist: „Gewißheiten“. Der Brief beginnt mit der Erinnerung an eine ergreifende Szene: „Klaras Erstkommunionfoto mit Sofortbildkamera kurz vor dem Kirchgang: während sie später mit brennender Kerze vor dem Altar stand, habe ich es mit nasser Hand in der Tasche zerknüllt" (339). Was vermag schon ein Foto wiederzugeben von der personalen Wirklichkeit eines solchen Augenblicks?

Dann stellt sich Rausak die Frage: „Was ist die Welt? Bilder entlang einer Bahnstrecke, Schnee auf Waldboden, Schafpferche, Hinterhöfe, Baustofflager? Gesammelte Gehaltsstreifen, Bilanzanalysen, uralte Gremialprotokolle, Aktenvermerke? Muffige Bürogänge, Konferenzluft, Polstertüren .. Motiv einer Sonate, Umrisse oft nachgelesener Denkgebäude? Oder bilden die Welt jene hundert eigenen Schlüsselerinnerungen jedes Lebenden vor und außer zufälligen Hüllen, die er pflichtgemäß ausfüllt?" (339).

Eine wichtige und grundlegende Frage: Von woher sollen wir die Welt anschauen? Sollen wir sie zu verstehen suchen von all dem, was da rein äußerlich geschieht und was Rausak in Stichworten aufzeigt? Oder ist all dieses Äußere dazu da, personales Erleben zu ermöglichen, Menschen anzuspornen, Personen zu formen auf den vielen Stationen eines irdischen Lebensweges?

Rausak nennt einige seiner Schlüsselerlebnisse, die er den Kindern aufheben will; darunter unter anderem dieses: „Wie eine Bergdohle vom Gipfel ab- 
hebt: stößt sich aufwärts, breitet die Flügel, schnellt über tausendmetrigen Steilabgrund hinaus, fällt, schwebt, kreist, segelt schließlich tief unter den Waldwipfeln: Eure Mutter Francine sagt: aus reiner Lebensfreude, denn hier oben gibt es keine Insekten zu jagen ... folgenloses Selbstzeugnis - Merkmal des Lebendigen, nirgendwo hingewandt, nicht Lockung, nicht Tarnung, nicht Arbeit - bloß schönes Muster, selig ausgefaltetes Nur-Dasein" (340).

Und dann stellt Rausak sich die Frage: „Wird es in deinem Leben genug gute Blicke geben, für dich, Kind, und für uns alle, völlig allein, von niemandem je angeschaut - also erschaffen, gehalten! - außer von Gott, dessen eigene Geschichte, dessen unbeendetes Abenteuer mit uns wir sind?" (341)

An dieser Stelle sei noch eine „biographische Zwischenbemerkung“ eingefügt. Rausak schreibt fast nirgends über seine Ehe. Und doch schimmert zwischen den Zeilen die tiefe Humanität seiner Beziehung zu Frau und Kindern hindurch. In der Beschreibung einer Szene, als er im Gedränge eines Tanzfestes zum Büfett gegangen ist, blitzt auf einmal etwas davon auf: „Als ich für Francine das Wiener Schnitzel durchs Gedränge rette, der Lichtblick: etwas in mir betet für dich, mehr kann mir niemand schenken" (56).

Und noch eine andere Stelle sei im gleichen Zusammenhang hier eingefügt. Rausak kommt von einer längeren Dienstreise zurück, steigt aus dem Zug und beschreibt das so: „Der Zug fährt in Stablau ein - Seehöhe $162 \mathrm{~m}$ gutmütige Arbeiter in Hemdsärmeln, Plastiktaschen voll schmutziger Monturen, müde Frauen, die nach der Arbeit noch Einkäufe erledigen, wortreiche Beständigkeit; abgemühte Angestellte schlürfen über Ölkies zum Einstieg, queren meinen Weg vom Trittbrett ... und dort, ja dort stehen die Meinen! Ihr habt mich abgeholt, Anna, Klara, Max an der Hand Francines.

Francines deutlich gewölbter Leib - für unseren neuen, jetzt schon geliebten kleinen Mitbewohner: Gottlieb oder Margarete ... In der diesjährigen Osternacht ist mir zur Taufwasserweihe - die ich schon diesem Ungeborenen gewidmet wußte, beim Halleluja die Stimme gebrochen.

Ihr seid mir über geleerte Bahnsteige lachend entgegengelaufen. Daß es solche Minuten gibt! Voll Dank, dies zu sehen. Dasein als Ort der Treue“ (351).

Daß es so etwas in heutiger Literatur, in einem Buch, das von der Kritik mehrfach auf das Niveau eines Musil oder Doderer gestellt wurde, gibt, ist für mich ein Zeichen der Hoffnung. Hier schreibt jemand in aller Freiheit gegen den Strom. Und er tut es mit überzeugender Kraft.

Mir scheint es bemerkenswert zu sein, daß dieses Buch schonungslos die Zerrissenheit unserer Welt, die Inhumanität mancher Strukturen, die entsetzlichen Folgen der Trägheit und Phantasielosigkeit bei den Verantwortungsträgern darstellt - und dann gleichwohl nicht in das heute so beliebte Klagelied über die "unheile Welt" einstimmt. Vielmehr werden wir auf einen Baum verwiesen, den auch wir in dieser unserer Gesellschaft "heilen" kön- 
nen: Den Raum der Beziehungen unter uns Menschen, die sich besonders in der Familie verdichten. Dieses Buch ist ebenso illusionslos der Realität unserer Welt gegenüber, wie ermutigend dem Leben gegenüber. Das ist wahrhaftig bemerkenswert in der Szene der Gegenwartsliteratur.

8. Gerne würde ich Ihnen die abschließenden drei Kapitel aus dem Buch "Stufen" noch vor Augen stellen. Das würde allerdings den Rahmen sprengen. Darum bitte ich Sie, sich mit einer kurzen Skizze zu begnügen.

Im ersten Kapitel mit der Überschrift „Der Fasan“ geht es um den teilweisen Zusammenbruch der Firma, die Problematik der Arbeitslosigkeit (das Buch ist 1979 erschienen) und um die menschlichen Probleme, die dahinterstehen. Es beschreibt eine leidenschaftliche, aber fair geführte Auseinandersetzung Rausaks mit einem Aufsichtsratsmitglied. Die Fehler des Managements werden schonungslos offengelegt.

Ich zitiere eine Bemerkung aus diesem Kapitel, die in der genannten Auseinandersetzung gefallen ist: „Es ist nicht Schuld, sondern Verdienst der Technik, daß sie heute als einzige Disziplin bindendes, brauchbares, weltweit anerkanntes Wissen erarbeitet hat. Würde nur mehr von ihren Grundsätzen auf das subjektive Geschluder anderer Bereiche angewandt. Würden den beredten Schreibtisch- und Podiumpult-Schwindlern doch ihre Tatfolgen so vorgerechnet wie dem Ingenieur, der sich bei einem Ventil irrt!" (365)

Das zweite Kapitel mit der Überschrift „Die Taube“ beschreibt den Zusammenbruch eines Teilwerks, um das der zuständige Betriebsdirektor bis zuletzt leidenschaftlich gekämpft hat. Von diesem Betriebsdirektor heißt es: „Dr. Leikermosers reine, persönlich integre Beschwörungen haben objektiv wie Revolten geklungen. Sie waren willkommenes Futter für Wochenblätter, die Korruption hören wollten, wenn er den Abstand zu soziotechnischen Visionen beklagte. Was wollte er konkret? Sachkundige, unser Spezialgebiet völlig erschließende Arbeit, auch als humane Leistung: enges, geradezu herzliches Zusammenrücken im Betrieb, allerfeinster Erfahrungsaustausch aus starkem, heißem Mitgefühl, dessen Mangel seiner Meinung nach in jene Versteinerung zwang, die uns heute lähme" (390).

Und dann heißt es weiter: „Leikermoser zerbrach wohl nicht an steter Widerlegung heiligmäßigen Lebensentwurfs, nicht an jener Ignoranz, die die Umwelt seinem innovativen Angebot gegenüberstellte, sondern an schneidend scharfer Einsicht der Unübertragbarkeit individuellen Betroffenseins, des derben Abstoßens nützlicher Vorschläge durch ein starres, stolzes Betriebssystem. Selbstlose, grundsätzliche Sicht gälte als zu vernachlässigende Subtilität, verlegen toleriertes Privathobby, unverbindliche Freizeitbeschäftigung, bar jeder Überzeugungskraft, wie sie etwa Mathematik genieße: daher marschiere die offizielle Halbwelt unbeeinflußbar mit endloser Blutspur in Sackgassen" (392).

Leikermoser ist zusammengebrochen und mußte in eine psychiatrische Klinik gebracht werden. 
All diese Sätze sind eingeflochten in den Gang durch die verlassenen Betriebshallen: Lebenswelt, wie wir sie alle kennen. Probleme, die jedem von uns schon bedrückend auf der Seele gelegen haben. Das ist das Faszinierende an diesem Buch, das ich ja nur in ganz kurzen Ausschnitten vorstellen kann.

Im dritten Kapitel der „Stufen“, überschrieben: „Der Kasuar“, wird erzählt, wie Rausak reagiert auf die Nachricht, daß er an Krebs erkrankt ist. Dieses Kapitel enthält noch einmal einen Rückblick, einen Überblick von einer früher so nicht erreichten Warte aus.

"Die schmerzlose Mitte der Sorgen ist erreicht. Rausak nimmt diesen Hohlraum - fast ein Gnadenort - tätig ein. Höchstmaß an Gleichgewichtssinn: diese Auszeichnung aller Vogelarten! Mitleid und Selbstschutz ausgewogen. Ein bis zur Grenze ausformuliertes Gewissen durchwirkt die Handlungen, beflügelt seine Arbeit ..." (415).

"Nun erst, im Hinblick der Grenze, kann unbehindert die aufgestaute, durch eigene Sorgen, Ängste verdrängte Kollegialität auswogen: keine Selbstbewahrung ist mehr geboten, die aus höchster Subjektivität ins Objektive umspringende Sicht, ein Strom tätiger, auch intellektuell produktiver Liebe bricht durch. Vor allem aber, sagt der Rausak bisher nie aufgefallene Belegschaftsrat Schupitt anläßlich seines Jubiläums, sind Sie, Herr Rausak, neben allem Fachlich-Guten, was meine Vorredner schon erwähnt haben, ein aufmerksamer, wirklich von allen Arbeitern in den Betrieben geschätzter Kollege! Rausak hierzu: Wenn ich unter allen natürlichen und juristischen Personen jene auswähle, die mein Leben mit nachhaltiger Wirkung mittrugen, so nimmt die EBC, trotz ihrer Aufspleißung in widersprüchliche Abläufe, den ersten Platz meiner Dankadressen ein“ (416).

Eine Kritik in "Christ und Gegenwart" (Nr. 21/1980) hat diesem Buch unter anderem vorgeworfen, daß sich aus ihm nicht eine Gestalt entwickle, an der sich „anschaulich verfolgbar und nicht nur in Splittern“ die evangelische Botschaft verwirkliche. Ich kann dieser Kritik nicht beipflichten. Mir ist diese Gestalt sehr deutlich geworden in dem Vater, den nichts interessiert als "gefundene Wahrheit zugunsten der Kinder" (16), und dem beim Anblick seiner Frau einmal durch den Kopf schießt: „Etwas in mir betet immer für dich" (56); in dem Ingenieur, der zur Großindustrie steht, weil es für das Überleben der Menschheit dazu keine Alternative gibt und gleichzeitig für die Vermenschlichung der industriellen Strukturen kämpft; in dem Verantwortungsträger, der den Traumtänzern gegenüber „eine verbindliche, außerhalb von uns wirksame, ohne Schäden nicht zu verbiegende Wahrheit" (266) reklamiert und deren Achtung fordert.

Zum Schluß sei noch etwas gesagt zu dem merkwürdigen Titel des Buches. Wer in der heutigen Welt unter einem solchen Anspruch leben will, wie Rausak es tut, der muß sich in vielfacher Hinsicht durchschlagen. Unsere Gesellschaftsstruktur, unsere Industriewelt und unsere verbürokratisierte Verwaltung sind einem Gestrüpp zu vergleichen, das zu bestehen man seine 
Stirn schon hart machen muß. Da sind dann die menschlichen Beziehungen in der Familie ein Ausgleich, ebenso wie die im Buch der „Preisungen“ geschilderten guten Erfahrungen; aber das Überwiegende ist ohne Zweifel das Gestrüpp. Dafür muß man sich in rechtverstandener Weise hart machen. Darum schreibt Rausak im ersten Brief an seine Kinder: „Liebe Kinder, lest euren ererbten Namen verkehrt: aus Rausak wird Kasuar: verborgenes Leben des Laufvogels im Dickicht! Ein Helm auf dem stets gesenkten Kopf zum Durchpflügen des Gestrüpps. Flügel zu knochenharten Kielen verwandelt: starkes Landtier, dennoch eine Flugseele" (324).

\section{SUMMARY: The „ostrich“ - a Christian in the industrial society}

The present text deals with the book „The Ostrich“ by Matthias Mander published in 1979 and wonders about the literary and social importance of this novel. ,The Ostrich is a bird which lives in the thicket and whose wings are therefore reorganized as rockhard quills: it is a strong land animal, yet a flier at heart'."

Matthias Mander uses this image to describe man in the industrial society. It is a book complete with a whole host of observations, thoughts, examples. It does not reveal its wealth until one has grasped its well-planned form and its structure. The numerous questions concerning a human organization of industrial society are brought up and discussed in an interesting literary form.

\section{RÉSUMÉ: Le „Casoar“, ou le chrétien dans la société industrielle}

Le texte présent s'intéresse au livre de Matthias Mander, „Le Casoar“, paru en 1979 et s'interroge sulla valeur littéraire et sociale de ce roman. „Le Casoar est un oiseau coureur qui vit dans les fourrés et dont les ailes sont, pour cette raison, formées de plumes avec un tuyau dur comme des os: un, animal terrestre fort - cependant une âme volante'."

C'est par cette image que Matthias Mander décrit l'homme dans la société industrielle. Un livre contenant une grande quantité d'observations, de raisonnements, d'exemples. Un livre qui n'ouvre ses richesses que quand on a compris sa forme bien réfléchie et sa structure. Les nombreuses questions relatives à une formation humainement équitable de la société industrielle sont soulevées et abordées sous une forme littéraire originale.

\section{RESUMEN: El „Casuario“ - un cristiano en la sociedad industrial}

Este texto se ocupa del libro „Der Kasuar“ de Matthias Mander, publicado en 1979, y se pregunta por el significado literário y social de esa novela. „El casuario es un ave corredora que vive entre los matorrales y que por eso sus alas se han transformado en plumas duras como huesos: ,un animal terrestre fuerte que posee, sin embargo, un alma voladora!"

Con esta imagen Matthias Mander describe al ser humano de la sociedad industrial. Un libro Ileno de observaciones, pensamientos, ejemplos. Muestra toda su riqueza solamente una vez que se ha entendido su muy bien pensada forma y estructura. Aquí se plantean numerosas preguntas referentes a la configuración humana de la sociedad industrial en una forma literaria muy original. 